\title{
Serum oxidizability potential is associated with age and exercise test results
}

\author{
Ehud Goldhammer • Yelena Rivlin • Sergei Shnizer • \\ Allah Shanati • Moran Sagiv • Uri Rosenschein
}

Received: 22 February 2010 / Accepted: 22 February 2010/Published online: 2 April 2010

(C) European Group for Research into Elderly and Physical Activity (EGREPA) 2010

\begin{abstract}
The purpose of this paper is to find out whether serum oxidizability potential measured before an exercise test (EXT) correlates with age and ischemic heart disease severity. Oxidizability potential was determined in 3 age groups, in gr. I patients $<45$ years, in gr. II age range $=45-70$ years, and in gr. III patients $>70$ years. Included subjects had chronic ischemic heart disease (IHD) and underwent a symptomlimited EXT upon initiation of a cardiac rehabilitation program. The thermo-chemiluminescence (TCL) assay was used to assess serum oxidizability potential. This assay is based on heat-induced oxidation of serum, leading to the formation of electronically excited species in the form of unstable carbonyls, which further decompose into stable carbonyls and light energy (low chemiluminescence). Measured photons emission is represented by a kinetic curve which is described by its amplitude and slope (=ratio). We assessed the correlations of TCL ratio with age, exercise duration, metabolic equivalents (METS), maximal heart rate (mHR), maximal systolic BP, $>1 \mathrm{~mm}$ S-T depression, diabetes, hypertension, smoking, left ventricular ejection
\end{abstract}

E. Goldhammer $\cdot$ Y. Rivlin $\cdot$ S. Shnizer $\cdot$ A. Shanati $\cdot$ M. Sagiv $\cdot$

U. Rosenschein

Department of Cardiology and Cardiac Rehabilitation,

Bnai Zion Medical Center, Faculty of Medicine, Technion,

Haifa 31048, Israel

E. Goldhammer • Y. Rivlin • S. Shnizer • A. Shanati • M. Sagiv •

U. Rosenschein

Zinman College of Physical Education,

Netanya, Israel

E. Goldhammer $(\square)$

Cardiac Rehabilitation, Bnai Zion Medical Center,

48, Golomb Str,

Haifa 31048, Israel

e-mail: egoldh@netvision.net.il fraction (LVEF) $>$ or $<40 \%$, previous myocardial infarction, and aorto-coronary bypass surgery. A high TCL ratio (\%) correlated well with METS $(r=0.82)$, mHR $(r=0.77)$ and with exercise-induced S-T segment shift $(r=0.86, p<0.05)$. A lower serum oxidizability potential, expressed as a low TCL ratio, thus suggestive of a previous high oxidative stress, was found in the two older age groups compared to gr. I ( $<45$ years), $p=0.041$, and in particular, in gr. III patients with low LVEF\%. The TCL ratio (\%) in gr. III was $188.7 \pm$ $14.5,192 \pm 17$ in gr. II, and $214 \pm 13$ in gr. I $(p<0.05)$, and was $166 \pm 13.1$ in gr. III with $\mathrm{LVEF}<45 \%$ as compared to $271 \pm 15.7$ in gr. I patients with $\mathrm{LVEF}>45 \%(p<0.01)$. A trend for lower TCL ratio (\%) was found in diabetic, hypertensive, and post-coronary bypass surgery patients. A paradoxically low TCL ratio (low oxidizability potential) was observed in patients without S-T depression compared to patients with S-T depression $(189 \pm 22$ vs. $201 \pm 15, p=$ NS), due to the fact these patients had a much lower LVEF\% and a lower exercise capacity. Serum oxidizability potential is associated with age, EXT parameters, results, and IHD severity. TCL ratio is an "easy-to-measure marker" that might be incorporated into risk assessment and prediction in aged IHD patients.

Keywords Oxidative stress - Exercise test . Ischemic heart disease

\section{Introduction}

Oxidative stress reflects a condition in which the balance between reactive oxygen species (ROS) production and the subsequent response of the antioxidant defense system is lost, becoming skewed in favor of free radical expression [1-3]. Although a multitude of free radicals exists (hydro- 
gen atoms, transition metal ions, carbon-centered radicals, sulfur-centered radicals, etc.), those derived from oxygen are referred to as ROS. ROS are highly reactive and very unstable molecules, which tend to initiate chain reactions resulting in irreversible chemical changes of lipids and proteins. These potentially deleterious reactions can result in profound cellular dysfunction and even cytotoxicity [4].

Aging is an inherently complex process that is manifested within an organism at genetic, molecular, cellular, organ, and system levels. Although the fundamental mechanisms are still poorly understood, a growing body of evidence points toward ROS as one of the primary determinants of aging. The "oxidative stress theory" holds that a progressive and irreversible accumulation of oxidative damage caused by ROS impacts on critical aspects of the aging process and contributes to impaired physiological function, increased incidence of disease, and a reduction in life span. While compelling correlative data have been generated to support the oxidative stress theory, a direct cause-and-effect relationship between the accumulation of oxidatively mediated damage and aging has not been strongly established $[5,6]$.

Recent data imply that measurement of oxidizability is a key to kinetic evaluation of oxidative processes of LDL, blood serum and other body fluids, and can be used for monitoring the oxidative stress in different diseases and antioxidant drug therapy [5-9]. The oxidizability of a biological sample is a measure of its susceptibility to oxidation. Growing evidence indicates that chronic and acute overproduction of ROS under pathophysiologic conditions is important for the development of cardiovascular diseases (CVD). ROS mediate various signaling pathways that underlie vascular inflammation in atherogenesis: from the initiation of fatty streak development through lesion progression to ultimate plaque rupture. Oxidative stress is the unifying mechanism for many CVD risk factors, which additionally supports its central role in CVD [10].

Evidence for increased oxidative stress has been found in plasma of patients with ischemic and nonischemic cardiomyopathy and correlates directly with the severity and chronicity of symptoms, and inversely with left ventricular ejection fraction (LVEF) [11, 12].

Free radical injury has also been implicated in the pathogenesis, evolution, and progression of heart failure [9, 13-15]. Furthermore, with the evolution of heart failure, there is a progressive increase in free radical injury and reduction of antioxidant reserves, which impacts significantly on prognosis.

Single bouts of aerobic and anaerobic exercise can induce an acute state of oxidative stress. This is indicated by an increased presence of oxidized molecules in a variety of tissues. Exercise mode, intensity and duration, as well as the kind of population under study, can impact on the extent of oxidation [16-22]. Exercise-induced oxidative stress has been investigated during and after exercise in chronic heart disease and chronic heart failure patients [13, 23, 24].

Most studies have shown an increased oxidative stress pre - and post-exercise However, no study has assessed the relationship between age, pre-exercise oxidizability potential, exercise test parameters, and EXT results.

\section{Materials and methods}

\section{Selection of patients}

Fifty-six chronic IHD patients, 13 females and 43 males, $11 \leq 45$ years (gr. I), 27 in age range $45-70$ years (gr. II), and $18 \geq 70$ years (gr. III). Forty-seven patients $(83.9 \%)$ had a previous myocardial infarction, $20(35.7 \%)$ had an aortocoronary bypass surgery (CABG), and 35 (62.5\%) had a previous percutaneous intervention (PCI). Eighteen patients (32.1\%) had diabetes mellitus (DM), 34 (60.7\%) hypertension, and $35(62.5 \%)$ dyslipidemia. Thirty-nine patients $(69.6 \%)$ were in New York Heart Association (NYHA) classes II-III, and 17 (30.3\%) in class I; patients in NYHA class IV were not included.

Twenty-nine patients $(51.7 \%)$ had $\mathrm{LVEF}<40 \%$, and 27 $(48.2 \%)$ had an ejection fraction (EF) $>40 \%$, including four patients who had normal EF ( $\geq 55 \%)$. Patients with an acute or recent febrile illness, significant liver dysfunction, or renal failure (serum creatinine $\geq 2.0 \mathrm{mgr} \%$ ) were excluded from the study (Table 1).

Subjects regularly using antioxidant supplements (vitamins A, C, E, or Co-Enzyme Q-10) or drugs with presumed antioxidant properties (statins, carvedilol) were required to stop these medications 7 days prior to the test.

All subjects underwent a symptom-limited EXT upon initiation of a cardiac rehabilitation program; prior to the EXT, a 2-mL venous blood sample was drawn for thermochemiluminescence (TCL) assay. Determination of TCL and oxidizability potential Photons emission during heating was measured by TCL analyzer (manufactured by Lumitest Ltd., Caesarea, Israel) using a photomultiplier model R265P (Hamamatsu Photonics Co. Ltd. Ichino-cho, Higashi-ku, Hamamatsu City, Japan) with a spectral response range of $280-650 \mathrm{~nm}$. The computer program of the device has two main functions: (1) analysis of the sample preparation $(0.05 \mathrm{~mL}$ of serum required for the test), (2) data processing, display, and storage. The serum under exam was spread over the surface of aluminum tray (a kind of miniature Petri dish) inside the sample preparation block and then was vacuum-dried. Then, the dish was mounted on a constant heater with heating temperature $80 \pm 0.5$ s.d. the analysis block and the photons emission was measured 
Table 1 Demographic and clinical data

\begin{tabular}{lccc}
\hline & Gr. I & Gr. II & Gr. III \\
\hline Patients No. & 11 & 27 & 18 \\
S-T depression $>1 \mathrm{~mm} \downarrow$ & $2(18.2 \%)$ & $19(70.4 \%)$ & $12(66.7 \%)$ \\
METS & $10.9 \pm 0.6$ & $7.4 \pm 0.5$ & $5.5 \pm 0.6$ \\
Exercise Duration (min) & $11.2 \pm 1.1$ & $7.5 \pm 0.8$ & $6.2 \pm 0.5$ \\
Max Systolic BP (mmHg) & $178 \pm 10$ & $147 \pm 11$ & $152 \pm 12$ \\
Max HR (bpm) & $159 \pm 11$ & $141 \pm 9$ & $127 \pm 6$ \\
F.C. I & $11(100 \%)$ & $3(15.8 \%)$ & $3(25 \%)$ \\
F.C. II-III & 0 & $16(84.2 \%)$ & $9(75 \%)$ \\
EF\% $<40$ & 0 & $17(62.9 \%)$ & $12(66.7 \%)$ \\
EF\%>40 & $11(100 \%)$ & $10(37.15)$ & $6(33.3 \%)$ \\
Previous M.I. & $4(36.4 \%)$ & $27(100 \%)$ & $16(88.9 \%)$ \\
Previous ACBG & $1(9.1 \%)$ & $15(55.6 \%)$ & $3(16.7 \%)$ \\
Previous PCI & $6(54.5 \%)$ & $24(88.9 \%)$ & $5(27.8 \%)$ \\
Dyslipidemia & $7(63.6 \%)$ & $17(62.9 \%)$ & $11(61.1 \%)$ \\
Current Smokers & $3(27.3 \%)$ & $9(33.3 \%)$ & $2(11.1 \%)$ \\
Diabetes & 0 & $9(33.3 \%)$ & $6(33.3 \%)$ \\
\hline
\end{tabular}

each second for 300 s. The obtained TCL curve was described mathematically as the amplitude of the kinetic curve of the photons emission and slope of the curve. The obtained curve is described mathematically as the amplitude of the kinetic curve and its slope (=ratio), which reflects the heat-induced susceptibility to oxidative modification of the tested sample, i.e., the residual oxidative capacity due to prior in vivo molecular oxidation. Thus, a lower curve slope suggests a lower oxidative potential, indicating higher oxidative activity before test.

\section{Statistical analysis}

Statistical analysis was performed using SPSS v15.0 (Chicago, IL, USA) and data were presented as means \pm SD. Student's $t$ test was used, due to the normal distribution of results, and correlations were determined by Pearson's coefficient. Associations were considered statistically significant when the $P$ value was $<0.05$. Regression analysis was performed in order to find out the independent variables with the most evident impact on TCL ratio.

\section{Results}

A lower serum oxidizability potential, expressed as a low TCL ratio, suggestive of a previous, high oxidative stress, was found in the older age groups (II and III) compared to the younger age group (I), $p<0.05$ and $p<0.01$.

The lowest values of TCL were found among gr. III patients with low LVEF\% (Fig. 1).

TCL ratio (\%) was $229 \pm 15$ in gr. I, $193 \pm 21$ in gr. II, and $188 \pm 14.7$ in gr. III, $(p<0.05$ and $p<0.01)$.
Gr. III patients with low LVEF $(<40 \%)$ had a TCL ratio of $177 \pm 12.1$ compared to $200 \pm 11.9$ in patients in the same group with a better LVEF ( $>40 \%), p<0.01$.

The TCL ratio correlated well with exercise tolerance expressed in metabolic equivalents as well with exercise duration ( $r=0.88$ and $0.91, p<0.01$, respectively). Similarly, TCL ratio correlated with exercise maximal heart rate $(r=0.79)$ and with exercise-induced $\geq 1 \mathrm{~mm}$ ST segment shift $(r=0.77)$. A trend for lower TCL ratio $(\%)$ was found in diabetic, hypertensive, and post-CABG patients $(193 \pm 14$, $194 \pm 17$, and $196 \pm 13$, respectively, $p=\mathrm{ns}$ ).

A paradoxically low TCL ratio (low oxidizability potential) was observed in patients without $\mathrm{S}-\mathrm{T}$ depression compared to patients with S-T depression (189 \pm 22 vs. $201 \pm$

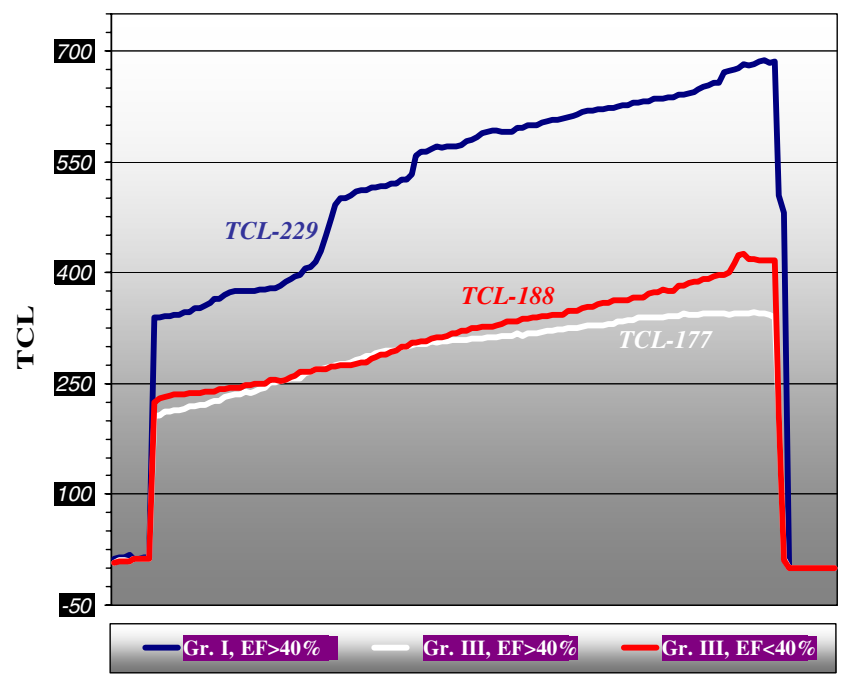

Fig. 1 TCL ratio in older patients with $L V E F \leq$ or $\geq 40 \%$ 
15, $p=\mathrm{ns}$ ), due to the fact that these patients had a much lower LVEF\% and a lower exercise capacity.

Regression analysis showed that older age $\geq 70$, and left ventricular $\mathrm{EF} \%$ were the best independent predictor of the TCL ratio $\left(R^{2}=0.87208, R=0.9338\right.$, St $\operatorname{Err}=17.76$, Adj $F=$ $0.87118, n=144, p<0.001)$.

\section{Discussion}

Reactive oxygen species and other oxidants are implicated in the mechanisms of biological aging and exercise-induced tissue damage.

The TCL assay used in our study is one among the accepted, validated, and reproducible methods [9-11] for measurement of oxidative stress and serum oxidizability potential. This assay is based on the heat-induced oxidation of a sample leading to the formation of electronically excited species (in particular of triplet excited carbonyls) and of light energy, low-level chemiluminescence.

Aging is associated with increased free radical generation in the skeletal muscle that can cause oxidative modification of protein, lipid, and DNA [5, 6, 25, 26]. Physical activity has many well-established health benefits, but strenuous exercise increases muscle oxygen flux and elicits intracellular events that can lead to increased oxidative injury. The paradox arises as to whether exercise would be advisable to aged population. Research evidence indicates that senescent organisms are more susceptible to oxidative stress during exercise because of the age-related ultrastructural and biochemical changes that facilitate formation of ROS.

Increased ROS production has been shown to impair endothelium-dependent vasorelaxation, to cause myocyte apoptosis, to increase monocyte adhesion, and inflammatory gene expression, thus contributing to myocardial and skeletal muscle contractile dysfunction and deterioration in congestive heart failure (CHF) patients [15].

Only a few studies have addressed the issue of oxidative stress in relation to age and exercise, and most of them were conducted on animals [27, 28].

In studies involving chronic IHD and CHF patients, a significant increase in exercise-induced plasma oxidative stress was found. Exercise mode, intensity, and duration, as well as the subject population tested, all can certainly impact the extent of oxidation [18-23, 29-31]. However, these studies disagree when pre-exercise oxidative status is examined. Sayar et al. [15] failed to find a significant difference in resting plasma oxidative stress in CHF patients as compared with controls, the likely reason for these unexpected findings being that the control group contained patients with many cardiovascular risk factors rather than healthy controls. Thus, it was suggested that the underlying risk factors may be associated with an increase in resting pre-exercise plasma malondialdehyde levels. Díaz-Vélez et al. [32] have reported similar findings concerning the resting plasma oxidative stress in symptomatic CHF patients $(\mathrm{LVEF}<40 \%)$, and in asymptomatic patients with LVEF $>40 \%$ without clinical evidence of CHF but with hypertension, DM or a history of myocardial infarction. On the other hand, in the study of Belch et al. [33], there was a significant, negative correlation between LVEF and oxidative stress.

No study so far has addressed the question whether the pre-exercise oxidative status is related to the age of the tested patients, and whether it may have an impact on EXT results, thus contributing to the risk assessment of these patients.

Our findings suggest that previous, chronic, or recurrent oxidative stress in older IHD patients practically reduces and depletes residual oxidative capacity. In other words, residual oxidative capacity is decreased due to prior recurrent in vivo molecular oxidation in these patients.

The study proves that the older the patient is, the sicker he is, with lower $\mathrm{EF} \%$, and lower exercise capacity, then, the lowest serum oxidizability potential, reflected by the lowest TCL ratio, is expected. Thus, assessment of TCL ratio at resting conditions may predict EXT results and, therefore, support risk assessment in chronic IHD patients according to their age group. Oxidizability potential assessment may have clinical applications in the routine follow-up of the atherosclerotic disease in elderly obese, diabetic, and hypertensive patients, as well as in elderly treated with drugs, vitamins, and food supplements with alleged or proved antiatherogenic or pro-atherogenic properties.

\section{References}

1. Shah AM, Channon KM (2004) Free radicals and redox signaling in cardiovascular disease. Heart 90:486-487

2. Becker LB (2004) New concepts in reactive oxygen species and cardiovascular reperfusion physiology. Cardiovasc Res 61:461470

3. Nordberg J, Arnér ES (2001) Reactive oxygen species, antioxidants, and the mammalian thioredoxin system. Free Radic Biol Med 31:1287-1312

4. Lefer DJ, Granger DN (2000) Oxidative stress and cardiac disease. Am J Med 109:315-323

5. Kregel KC, Zhang HZ (2007) An integrated view of oxidative stress in aging: basic mechanisms, functional effects, and pathological considerations. Am J Physiol Regul Integr Comp Physiol 292:18-36

6. Buffenstein R, Edrey YH, Yang T, Mele J (2008) The oxidative stress theory of aging: embattled or invincible? Insights from nontraditional model organisms. AGE 30(2-3):99-109

7. Kontush A, Beisiegel U (1999) Measurement of oxidizability of blood plasma. Methods Enzymol 299:35-49

8. Haffner SM, Agil A, Mykkanen L, Stern MP, Jialal I (1995) Plasma oxidizability in subjects with normal glucose tolerance, 
impaired glucose tolerance, and NIDDM. Diabetes Care 18:646653

9. Friedman J, Peleg E, Kagan T, Shnizer S, Rosenthal T (2003) Oxidative stress in hypertensive, diabetic, and diabetic hypertensive rats. Am J Hypertens 16:1049-1052

10. Goldhammer E, Maor I, Shnitzer S, Lanir A, Abinader EG (2007) The early anti-oxidant effect of carvedilol predicts the clinical course in congestive heart failure patients. J Cardiovasc Med (Hagerstown) 8:453-456

11. Amir O, Paz H, Rogowski O, Barshai M, Sagiv M, Shnizer S, Reznick AZ, Amir RE (2009) Serum oxidative stress level correlates with clinical parameters in chronic systolic heart failure patients. Clin Cardiol 32:199-203

12. Madamanchi NR, Vendrov A, Runge MS (2005) Oxidative stress and vascular disease. Arterioscler Thromb Vasc Biol 25:29-38

13. Harrison D, Griendling KK, Landmesser U, Hornig B, Drexler H (2003) Role of oxidative stress in atherosclerosis. Am J Cardiol 91:7A-11A

14. Vassalle C, Petrozzi L, Botto N, Andreassi MG, Zucchelli GC (2004) Oxidative stress and its association with coronary artery disease and different atherogenic risk factors. J Intern Med 256:308-315

15. Sayar N, Terzi S, Yilmaz HY, Tangurek B, Bilsel T, Cakmak N, Orhan L, Emre A, Ciloglu F, Peker I, Yesilcimen K (2007) Exercise-induced increase in lipid peroxidation in patients with chronic heart failure: relation to exercise intolerance. Cardiology 108:307-313

16. Giordano FJ (2005) Oxygen, oxidative stress, hypoxia, and heart failure. J Clin Invest 115:500-508

17. Keith M, Geranmayegan A, Sole MJ, Kurian R, Robinson A, Omran AS, Jeejeebhoy KN (1998) Increased oxidative stress in patients with congestive heart failure. J Am Coll Cardiol 31:1352-1356

18. Mak S, Newton GE (2001) The oxidative stress hypothesis of congestive heart failure: radical thoughts. Chest 120:2035-2046

19. Groussard C, Rannou-Bekono F, Machefer G, Chevanne M, Vincent S, Sergent O, Cillard J, Gratas-Delamarche A (2003) Changes in blood lipid peroxidation markers and antioxidants after a single sprint anaerobic exercise. Eur J Appl Physiol 89:1420

20. Goto C, Higashi Y, Kimura M, Noma K, Hara K, Nakagawa K, Kawamura M, Chayama K, Yoshizumi M, Nara I (2003) Effect of different intensities of exercise on endothelium-dependent nitric oxide and oxidative stress. Circulation 108:530-535

21. Knez WL, Jenkins DG, Coombes JS (2007) Oxidative stress in half and full Ironman triathletes. Med Sci Sports Exerc 39:283288

22. Nikolaidis MG, Kyparos A, Hadziioannou M, Panou N, Samaras L, Jamurtas AZ, Kouretas D (2007) Acute exercise markedly increases blood oxidative stress in boys and girls. Appl Physiol Nutr Metab 32:197-205

23. Fisher-Wellman K, Bloomer RJ (2009) Acute exercise and oxidative stress: a 30 year history. Dyn Med 8:1

24. Meijer EP, Goris AH, van Dongen JL, Bast A, Westerterp KR (2002) Exercise-induced oxidative stress in older adults as a function of habitual activity level. J Am Geriatr Soc 50:349-353

25. Ames BN, Shigenaga MK, Hagen TM (1993) Oxidants, antioxidants and the degenerative diseases of ageing. Proc Natl Acad Sci 90:7915-7922

26. Berlett BS, Stadman ER (1997) Protein oxidation in aging, disease and oxidative stress. J Biol Chem 272(-):20313-20316

27. Ji LL (2001) Exercise at old age: does it increase or alleviate oxidative stress? Ann N Y Acad Sci 928:236-247

28. Bejma J, Ramires P, Ji LL (2000) Free radical generation and oxidative stress with ageing and exercise: differential effects in the myocardium and liver. Acta Physiol Scand 169(4):343-351

29. Nishiyama $Y$, Ikeda $H$, Haramaki N, Yoshida N, Imaizumi $T$ (1998) Oxidative stress is related to exercise intolerance in patients with heart failure. Am Heart J 135:115-120

30. Andican G, Koldaș L, Seven A, Ayan F, Sirmaci N, Burçak G (2001) Biochemical evaluation of oxidative stress during exercise in patients with coronary heart disease. Clin Chem Lab Med 39:234-238

31. Lo Presti R, D'Amico T, Montana M, Canino B, Amodeo G, Ciancarelli MG, Caimi G (2007) Evaluation of oxidative status in coronary heart disease at baseline and during exercise test. Clin Hemorheol Microcirc 37:339-345

32. Díaz-Vélez CR, García-Castiñeiras S, Mendoza-Ramos E, HernándezLópez E (1996) Increased malondialdehyde in peripheral blood of patients with congestive heart failure. Am Heart J 131:146152

33. Belch JJ, Bridges AB, Scott N, Chopra M (1991) Oxygen free radicals and congestive heart failure. Br Heart J 65:245-248 